\title{
THE FORTY-EIGHTH ANNUAL MEETING OF THE AMERICAN ASSOCIATION FOR THE ADVANCEMENT OF SCIENCE.
}

The forty-eighth annual meeting of the American Association for the Advancement of Science was held in Columbus, Ohio, August 21-26. There were 352 members and associates in attendance and 273 papers were presented before the sections. The members came from twenty-eight different states, the District of Columbia, and Canada. A considerable number of members of affiliated societies were also in attendance, many of whom took part in the proceedings of the sections in which they were interested. Their presence increased the general scientific interest; and in view of the desire of the various sections to have still closer relations with the respective affiliated societies, authority was given to the officers of the Association whereby any section may arrange for a joint meeting with societies of a similar scope. It is hoped that the AMERIcan Mathematical Society and the Society for the Promotion of Engineering Education may meet next year with Sections A and D respectively. Probably several other societies will hold joint meetings with the corresponding sections of the Association.

The next meeting of the Association will be held in New York City during the last week of June, 1900, under the presidency of Professor R. S. Woodward, Columbia University. Professor Asaph Hall, Jr., Michigan University, will be vice-president of Section A, and Dr. W. M. Strong, Yale University, will be secretary. It is hoped that the change in the time of these meetings from the latter part of August to the last week in June will tend to increase the attendance. This change seemed especially desirable for next year since it will enable many to attend the meetings of the Association on their way to the Paris exposition.

The meetings of the section of mathematics and astronomy were well attended. The officers of this section were: vice-president, Alexander Macfarlane; secretary, J. F. Hayford ; councilor, C. L. Doolittle; sectional committee, Alexander Macfarlane, J. F. Hayford, G. B. Halsted, R. D. Bohannan, W. M. Strong. The Council elected the following mathematicians and astronomers to fellowship in the Association : S. M. Barton, W. P. Durfee, O. L. Fassig, R. A. Fessenden, C. E. Furness, R. A. Harris, G. A. Hill, 
J. E. Keeler, W. H. Metzler, G. W. Meyers, D. A. Murray, W. M. Strong, J. H. Tanner, A. G. Webster.

The opening address of the Vice-President of Section A was on "The fundamental principles of algebra." It has been published in Science, September 15, 1899. The following papers were presented at the meetings of this section :

(1) Professor G. B. Halsted : "Report on progress in non-euclidean geometry."

(2) Professor L. E. Dickson: "Report on progress in the theory of linear groups."

(3) Professor Asaph Hall, Jr.: "The aberration constant from observations of Polaris." "

(4) Professor R. W. McFarlane : "Ancient eclipses and chronology."

(5) Professor H. C. Lond : "Some points in the design of a spectroscope."

(6) Professor J. V. Collins: "Note on Grassmann's proof that there can be but two kinds of lineal multiplication of two factors."

(7) Professor G. J. Stokes : "The theory of mathematical inference."

(8) Mr. J. W. DAvis: "Internal forces that generate stellar atmospheres."

(9) Professor R. A. Fessenden : "The determination of the nature of electricity and magnetism, including a determination of the density of the ether."

(10) Dr. G. A. Mrller: "On the commutators of a group."

(11) Dr. S. Kimura : "Linear vector functions."

Three of the titles that appeared on the programme are not reproduced here on account of the fact that these papers did not reach the secretary in time to be presented at the meeting. In the absence of the authors the papers by Professor Stokes and Dr. Kimura were presented by Professors Macfarlane and Shaw respectively. The paper by Professor Fessenden was read before a joint session of Sections $A$ and $B$ and will be reviewed in connection with the papers of Section B. Professor Dickson's report has already appeared in the October number of the Bullwatin. Dr. Miller's paper will appear in a later number. Abstracts of the other papers are given below.

Professor Halsted contrasted the neglect of Lobachévski and Bolyai with the present interest in non-euclidean geometry as exhibited by the appearance in the Monist of a long article on this subject by Poincaré. The investigations 
of Sophus Lie and the contributions of Gérard were discussed. Burnside's articles in the Proceedings of the London Mathematical Society were interpreted. The new book, Universal algebra, by Whitehead, and the important discoveries freeing us from the misleading conceptions of curvature of space in non-euclidean geometry were elucidated. The philosophic bearings of the new space ideas were illustrated with special reference to B. A. W. Russell's book. Killing and Clifford spaces were noted. The reproduction of the non-euclidean classics, especially by Engel and Stäckel, was described, also the work of the Hungarian academy of sciences. The history of the subject (Loria) and finally the bibliography closed the report, which has recently appeared in Science, October 20, 1899.

Professor Hall stated that observations of the zenith distances of Polaris for the determination of the aberration constant have been made with the meridian circle of the Detroit Observatory since about May 1, 1898. The direct observations made during the interval from May, 1898 to July, 1899, have been roughly reduced and give the values $20 . " 60$ and 20." 58 , from pointings above and below the pole respectively. These seem rather large, the correct value being supposed to be about $20 .^{\prime \prime} 50$. By observing above and below the pole the latitude variation is eliminated, but the effect of temperature on the instrumental readings needs to be followed very carefully. It is to be hoped that this work can be kept up through the year 1900. It has been suggested by several astronomers and physicists that a correct theoretical treatment of aberration has not yet been obtained. The values of the aberration constant recently found vary a great deal, ranging from about $20 . .^{\prime \prime} 31$ to 20."66. An increase in the value of this constant would indicate, of course, a decrease in the usually adopted value of the solar parallax.

The burden of the argument in Professor McFarlane's paper was that eclipses have not aided in fixing any historical event in its proper place in history ; that the difficulty is not mathematical, but arises from want of exactness in historical records ; and that this want of exactness arose from the want of a fixed time from which to reckon, because the writers of ancient histories, so-called, gave, in general, no dates at all. Moreover, all references to eclipses are vague in the extreme. 'Taking the most celebrated of all the cases-the eclipse of Thales-it was 
shown that Sir George Airy and Mr. Baily differed about 25 years in the date of the eclipse, and that other astronomers, and celebrated' scholars, in England, France, Germany, and elsewhere, have set the time in nine different years scattered over a period of more than forty-six years. If the most celebrated case thus falls, there is no standing for the others.

At one place in the demonstration given on page 28 of the Ausdehnungslehre of 1862 , an equation can be derived from the preceding in a somewhat different manner than that given. As this theorem is fundamental in Grassman's system, Dr. Collins worked out the following alternative proof:

In the derivation of equation (c) from

$$
\Sigma x_{r, u} x_{s, v}\left(\alpha_{r, s}\left[e_{u} e_{v}\right]+\alpha_{s, r}\left[e_{v} e_{u}\right]\right)=0
$$

by successive substitution of $2 x_{a, c}$ and $x_{a, c}$ instead of +1 and -1 as values of a particular $x_{r, u}$, we get

$$
\Sigma x_{s, v}\left(\alpha_{a, s}\left[e_{c} e_{v}\right]+\alpha_{s, a}\left[e_{v} e_{c}\right]\right)+3 x_{a, c_{a, a}}\left[e_{a} e_{a}\right]=0,
$$

in which the summation does not extend to $s=a, v=c$ at the same time. Inserting the missing term,

$$
\Sigma x_{s, v}\left(\alpha_{a, s}\left[e_{c} e_{v}\right]+\alpha_{s, a}\left[e_{v} e_{c}\right]\right)+2 x_{a, c} \alpha_{a, a}\left[e_{a} e_{a}\right]=0 .
$$

By putting a particular $x_{s, v}$, as $x_{b, d}$ first equal to 2 and then equal to 1 , we have finally

$$
a_{a, b}\left[e_{c} e_{d}\right]+\alpha_{b, a}\left[e_{d} e_{c}\right]=0 .
$$

The derivation, by symmetry, of equation (b) from the equation which precedes it

$$
\Sigma x_{r, u} x_{s, v} \alpha_{r, s}\left[e_{u} e_{v}\right]=0
$$

is very interesting on account of its simplicity, but suggests logical doubts. Mr. Collins points out that the terms of

$$
\Sigma x_{r, u} x_{s, v} \alpha_{r, s}\left[e_{u} e_{v}\right]
$$

which have the same coefficients (products of $x$ 's) are of two types, viz.,

$$
x_{r, u} x_{s, v}\left(\alpha_{r, \varepsilon}\left[e_{u} e_{v}\right]+\alpha_{s, r}\left[e_{v} c_{u}\right]\right), \quad x_{r, u_{r, u}} x_{r, r}\left[e_{u} e_{u}\right]
$$

whose combination, in view of

$$
\Sigma x_{r, u_{s, v}} \alpha_{v, s}\left[e_{u} e_{v}\right]=0,
$$


gives (b) at once. Thus the direct derivation gives the same result as symmetry.

The paper of Professor Stokes, who occupies the chair of logic in Queens College, Cork, compared the fundamental equations $x^{2}=x$ and $x(1-x)=0$ of Boole's calculus with the fundamental equations $l_{r}^{2}=0$ and $l_{r} l_{s}=-l_{s} l_{r}$ of Grassmann's calculus of extension, and stated :- "Whereas the first set of equations belong to an algebra of self-identical unrelated units, the second set belong to an algebra in which relations, synthesis, reference beyond self, is essential."

In a paper on the " Imaginary of logic" before the British Association in 1898 the author put forward the view that as the square root of a positive quantity is + or - , the square root of a negative quantity may be expected to be + and - , in view of the logical relation between and and or pointed out originally by DeMorgan, and subsequently by Schroeder. $\mathrm{He}$ considers that this view receives support from the paper of Kempe on the theory of mathematical form. He concludes as follows:- "the fertile proportions of mathematics from which its wealth of content and the treasures of mathematical knowledge are drawn, are not synthetical in the sense in which Kant and the empiricists alike maintain them to be, viz : that the truths preëxist and are then seen to be synthetical, the synthetical character being as it were something subsequent to the content of the proportions and attached to it as it were adjectively; but in this sense that those proportions are themselves the product of pure synthesis, that the very possibility of advance from entity to entity, unit to unit, or relation to correlate, determines all those laws which mathematics is employed in exploring and tracing into all their consequences, and which are infinitely more fruitful than the analytical laws of formal logic or the calculus of classes and statements. Pure synthesis generally is that ' necessity of matter' of which Hamilton spoke; the principle of material consequences which characterizes every genuine department of mathematics and defies further logical analysis."

The conclusion reached in the paper by Mr. Davis is that every celestial body wholly gaseous, large or small, composed of a mixture in which the proportion of heavier gases is considerable, necessarily generates an outrushing atmosphere, and that this atmosphere ceases to exist only upon the exhaustion of the lighter gases or the liquefaction of the central core. The atmosphere is exceedingly tenuous. 
In case of the sun the yearly shrinkage of the nucleus is known to be only a few hundred feet. The consequent extrusion of the lighter gases annually adds to the atmosphere a few thousand feet, and this in the course of the year is spread throughout the solar system.

Dr. Kimura's paper is a development of many formulæ for the application of the nonion operator $\varphi$ and its differential nabla $\phi C$ to mathematical physics. Expressions were given for the moduli of the characteristic equations of powers of $\varphi$; expressions for powers of $\varphi$ in terms of any three other powers of $\varphi$; expressions for the reduction of products such as $\varphi^{\prime p} \varphi^{q}$ or $\varphi^{q} \varphi^{\prime p}$, where $\varphi^{\prime}$ is the conjugate of $\varphi$; expressions involving the self-conjugate part of $\varphi$, and the rotation-vector of $\varphi$; and finally a considerable study of the operator

$$
{ }_{\phi} C=\left|\begin{array}{lll}
\frac{\partial}{\partial a_{1}} & \frac{\partial}{\partial b_{1}} & \frac{\partial}{\partial c_{1}} \\
\frac{\partial}{\partial \alpha_{2}} & \frac{\partial}{\partial b_{2}} & \frac{\partial}{\partial c_{2}} \\
\frac{\partial}{\partial \alpha_{3}} & \frac{\partial}{\partial b_{3}} & \frac{\partial}{\partial c_{3}}
\end{array}\right| \varphi, \text { where } \varphi=\left(\begin{array}{l}
\left.a_{1} b_{1} c_{1}\right) \\
a_{2} b_{2} c_{2} \\
a_{3} b_{3} c_{3}
\end{array} \mid\right.
$$

It is proper to add that the formulæ in the main are applicable only to linear vector operators which have: (a) distinct roots. (b) two equal roots, but reducible to the form

$$
\varphi=-\frac{g_{1} \rho_{1} s \rho_{2} \rho_{8}()-g_{1} \rho_{2} s \rho_{3} \rho_{1}()-g_{2} \rho_{8} s \rho_{1} \rho_{2}()}{s_{1} \rho_{1} \rho_{2} \rho_{3}} .
$$

(c) three equal roots, but reducible to the form $\varphi=g$.

CoRnell University.

G. A. Miller. 